\title{
Multi-Channel Preemptive Priority Model for Spectrum Mobility in Cognitive Radio Networks
}

\author{
S. E. Saad', I. F. Tarrad', A. A. Ammar \\ ${ }^{1}$ Departement of Electronics and Communications Engineering, High Institute for Engineering and Technology, Egypt \\ ${ }^{2,3}$ Electrical Engineering Department, Faculty of Engineering, Al-Azhar University, Egypt
}

\begin{tabular}{|c|c|}
\hline Article Info & ABSTRACT \\
\hline & \multirow{10}{*}{$\begin{array}{l}\text { Cognitive Radio techniques have been proposed for improving utilization of } \\
\text { the spectrum by exploiting the unoccupied bands of the licensed spectrum. } \\
\text { This paper proposes a preemptive multi-channel access model for prioritized } \\
\text { cognitive radio networks using an iterative method of queuing theory to solve } \\
\text { the spectrum scarcity problem. The proposed model formulates accurate } \\
\text { closed form of an expected waiting time in the queue, an expected number of } \\
\text { users in the queue, an expected waiting time in the system, and an expected } \\
\text { number of users in the system. The results compared to the basic model } \\
\text { (without preemptive priority) show that, the waiting time in queue and the } \\
\text { waiting time in the system compared to the basic model will be improved by } \\
92.99 \% \text { and } 33.15 \% \text { respectively for class one secondary user. The results } \\
\text { also show that, the waiting time in queue and the waiting time in the system } \\
\text { will be improved by } 43.25 \% \text { and } 15.42 \% \text { respectively for class two } \\
\text { secondary users. The proposed model investigates the desirable schedules of } \\
\text { primary and secondary users. }\end{array}$} \\
\hline Received Mar 2, 2018 & \\
\hline Revised Jul 24, 2018 & \\
\hline Accepted Aug 11, 2018 & \\
\hline Keyword: & \\
\hline Cognitive Radio & \\
\hline Multi-Channel & \\
\hline Preemptive Priority & \\
\hline Queuing Theory & \\
\hline Spectrum Mobility & \\
\hline
\end{tabular}

Copyright $\left({ }_{0}\right.$ 2018Institute of Advanced Engineering and Science. All rights reserved.

\section{Corresponding Author:}

\section{S. E. Saad,}

Departement of Electronics and Communications Engineering,

High Institute for Engineering and Technology, Al-Obour,

Kilo 21 Cairo / Belbies Rd, Egypt-P.O. Box 27 Obour City, Egypt.

Email: s.elsayed8585@gmail.com

\section{INTRODUCTION}

During the last decade, the wireless communications have been developed. The actual spectral occupancy over some frequency bands studied was found to be virtually empty. In O.W. Bello et al authors carried out spectrum measurement in urban and rural locations, covering bands of $50 \mathrm{MHz}$ and $6 \mathrm{GHz}$ [1]. The results show that, the average spectral occupancy of $5.08 \%$ and $0.18 \%$ in urban and rural locations respectively during weekdays and $1.45 \%$ on weekends for urban locations. So, the limited available spectrum and the inefficiency using of the spectrum have become one of the current problems in wireless communications. Cognitive radio technique has been proposed as a solution to these problems.

The cognitive radio technology is based on the opportunistic usage of the spectrum by allowing unlicensed users to exploit frequency bands of licensed users. Opportunistic spectrum access involves two tasks of cognitive radio system: spectrum sensing and dynamic spectrum access.

Many spectrum sensing techniques have been recently studied, namely Energy detection [2], hybrid spectrum sensing method [3], discrete markov chain based method [4], eigen value based spectrum sensing [5], and matched filter detector and cyclostationary detector [6]-[7].

Many dynamic spectrum access models have been studied. In L. Chen et al the authors proposed M/M/1 queue which is represented by a two dimensional state transition graph [8]. The authors proposed a queuing model for heterogeneous data transmissions in underlay cognitive radio networks. In this model, 
safety or emergency related messages possess preemptive higher priority over nonemergency messages. This model has two classes; high priority for primary user and low priority for secondary user.

In M.E. Bayrakdar and A. Çalhan non-preemptive M/G/1 priority queuing model of spectrum hand-off scheme was proposed in cognitive radio networks [9-10]. In T.C. Chu et al, the authors proposed a Dynamic Spectrum Access scheme for cognitive radio networks, where priorities for the bandwidth, the spectrum access, and spectrum hand-off are considered for three types of traffics [11]. Paper has adopted a multidimensional Markov chain with three state variables to analyze the state transitions of the dynamic spectrum access scheme which enables us to obtain the steady state distribution of the number of each kind of traffics in the system.

It is very difficult to analyze the behavior of multi-channel of the priority queue model when channel usage by primary and secondary users [12]. Although the vast research on the analysis of priority queues with a single channel facility, efforts on the characterization of multi channel queues were not as extensive or fruitful due to their complications. This is essentially unfortunate since the modeling of many telecommunications problems can be suitably placed in the framework of a multi channel system, such as multi channel cognitive radio networks [13].

In this paper, multi-channel preemptive priority model based on queuing theory has been proposed as a solution to this problem. The main contributions of this paper are as follows:

1. Queuing based spectrum access scheme for prioritized cognitive radio networks is proposed.

2. An accurate analytical model for spectrum access queuing based scheme priority service discipline is derived.

3. The proposed model can be applied for multi-priority secondary user.

4. The proposed model can be generalized for multi-channels.

The rest of this paper is organized as follows: In Section 2, the proposed model has been introduced. The simulation results of the proposed model have been discussed in Section 3. Finally, Section 4 presents the conclusion of the proposed model.

\section{PROPOSED MODEL}

In this section, a proposed model based on queuing theory has been presented. Symbols and notations used in this paper are listed in Table 1.

Table 1. The Description of Symbols

\begin{tabular}{cl}
\hline Symbol & \\
\hline$\lambda_{\mathrm{p}}$ & Mean arrival rate of the primary user \\
$\lambda_{\mathrm{si}}$ & Mean arrival rate of class i secondary user \\
$\lambda$ & Mean arrival rate of all users \\
$\mu$ & mean service rate \\
$\rho$ & Utilization factor it's $=\lambda / \mathrm{C} \mu$ \\
$\mathrm{C}$ & number of channels in the system \\
$P_{n}$ & probability of exactly n users in the system \\
$L_{s}$ & Average number of users in the system \\
$L_{q}$ & Average number of users in the waiting buffer \\
$W_{p}$ & Average waiting time of the primary users in the system \\
$W_{\mathrm{si}}$ & Average waiting time of users in the system \\
$W_{q}$ & Average waiting time of users in buffer \\
\hline
\end{tabular}

\subsection{Primary User Model}

In this model, there are several channels used by primary users and can only be used by secondary users when the channels are free. There are three possible cases; the arrived primary user may be encounter:

1. There is an empty channel so; the primary user will be accessed.

2. There isn't an empty channel and secondary user in service so; the secondary user will be ejected to waiting buffer and primary user accessed.

3. There is neither an empty channel nor secondary user in service so; the primary user enters the queue. 
Figure 1. Primary User Model

Figure 1 represents the possible three cases when the primary user has arrived.

\subsection{Secondary User Model}

The secondary users keep searching for channels that are free (available for use) at some points in time. There are three possible cases; the secondary user may be encounter:

1. There is an empty channel so; the secondary user will be accessed.

2. There isn't an empty channel and there is lower priority secondary user in service so; the lower priority secondary user will be ejected to waiting buffer and the higher priority user will be accessed.

3. There is neither an empty channel nor lower priority secondary user in service so; the arrived secondary user enters the queue. 
Figure 2 shows the possible three cases; the secondary user may be encounter. Users are selected to begin service in the order of their priority classes, but on a first come first served basis within each class. There are two basic priority discipline models, preemptive priorities and non-preemptive priorities. Preemptive priorities mean that, the lowest priority user being served is preempted whenever a higher priority user enters the system. Non-preemptive priorities, a user being served cannot be preempted if a higher priority user enters the queueing system. In this paper, the preemptive priority model has been selected.

\subsection{Analytical Model}

In this model, assume that; the arriving users and leaving users of the queuing system occur according to the birth and death process. However, the term birth refers to the arrival of a new user into the system, and death refers to the departure of a served user.

The solution has the following steps according to the general method described in [14]:

1. Obtain the steady state equations governing the queue.

2. Solve the equations for finding out the probability distribution of queue length by: a) Iterative method. b) Using generating functions. c) Using linear operators.

3. Obtain formula for $\mathrm{Ls}, \mathrm{Lq}, \mathrm{Ws}$ and, $\mathrm{Wq}$ as shown in Table 1.

After constructing the balance equations for all the states in terms of the $P_{n}$ probabilities, this system of equations can be solved.

By Applying this procedure yields

$$
P_{n}=\left\{\begin{array}{lr}
\frac{1}{n !} \cdot\left(\frac{\lambda}{\mu}\right)^{n} P_{0} \quad \text { for } & 0 \leq n<C \\
\frac{1}{C ! \cdot C^{n-C} \cdot\left(\frac{\lambda}{\mu}\right)^{n} P_{0} \text { for }} & n \geq C
\end{array}\right.
$$

A queueing model is based on the birth and death process, so the state of the system $\mathrm{n}$ represents the number of users in the queueing system, the key measures of performance for the queueing system (L, Lq, $\mathrm{W}$, and $\mathrm{Wq}$ ) can be obtained.

From definition of $L_{q}$

$$
L_{q}=\sum_{n=C}^{\infty}(n-C) P_{n}
$$

By solving this equation we get

$$
L_{q}=\frac{1}{C \cdot C !} \cdot \frac{(C \rho)^{C+1}}{(1-\rho)^{2}} \cdot P_{0}
$$

It has been proved that in a steady state queuing system,

$$
\mathrm{L}=\lambda \mathrm{W}
$$

This equation is called Little's formula.

Assume that the mean service time is a constant, $\frac{1}{\mu}$. It then follows that

$$
\mathrm{W}=\mathrm{W}_{\mathrm{q}}+\frac{1}{\mu}
$$

These relationships are extremely important because they enable the fundamental parameters Lq, $\mathrm{Wq}, \mathrm{L}$, and $\mathrm{W}$ to be immediately determined as soon as one is found analytically. This relation is fortunate because some of these quantities often are much easier to find than others when a queuing model is solved from basic principles.

\subsection{The Average Waiting Time in the System}

For Preemptive priority, waiting time for primary users must equal waiting time for the corresponding one class model. Because the waiting times for primary users are completely unaffected by the 
presence of users in the secondary classes, waiting time will be the same for any other values of arrival rates , including $\lambda_{\mathrm{si}}=0$. The waiting time for primary user can be formulated from Equations (3), (4), and (5):

$$
\mathrm{W}_{\mathrm{p}}=\frac{1}{\left[\mathrm{c} ! \frac{(\mathrm{c} \mu-\lambda)}{\left(\frac{\lambda}{\mu}\right)^{\mathrm{c}}} \sum_{\mathrm{n}=0}^{\mathrm{c}-1} \frac{\left(\frac{\lambda}{\mu}\right)^{\mathrm{n}}}{\mathrm{n} !}+\mathrm{c} \mu\right]\left[1-\frac{\lambda_{\mathrm{p}}}{\mathrm{c} \mu}\right]}+\frac{1}{\mu}
$$

Waiting time for secondary user can be calculated by an iterative procedure.

Class one secondary user is completely unaffected by lower-priority classes, which can therefore be ignored in the analysis. Let $\mathrm{W}_{\mathrm{X} 1}$ be the expected waiting time in the system of a random arrival in either of these primary users and class one secondary users:

$$
\begin{aligned}
& \mathrm{W}_{\mathrm{x} 1}=\frac{1}{\left[\mathrm{c} ! \frac{\left(\mathrm{c} \mu-\lambda_{\mathrm{x} 1}\right)}{\left(\frac{\lambda_{\mathrm{x} 1}}{\mu}\right)^{\mathrm{c}}} \sum_{\mathrm{n}=0}^{\mathrm{c}-1} \frac{\left(\frac{\lambda_{\mathrm{x} 1}}{\mu}\right)^{\mathrm{n}}}{\mathrm{n} !}+\mathrm{c} \mu\right]\left[1-\frac{\lambda_{\mathrm{x} 1}}{\mathrm{c} \mu}\right]}+\frac{1}{\mu} \\
& \lambda_{\mathrm{x} 1}=\lambda_{\mathrm{p}}+\lambda_{\mathrm{s} 1}
\end{aligned}
$$

So the probability is that this arrival is primary user $\frac{\lambda_{\mathrm{p}}}{\lambda_{\mathrm{p}}+\lambda_{\mathrm{s} 1}}$ and $\frac{\lambda_{\mathrm{s} 1}}{\lambda_{\mathrm{p}}+\lambda_{\mathrm{s} 1}}$, that it is in class one secondary user. Therefore, $\mathrm{W}_{\mathrm{x} 1}=\frac{\lambda_{\mathrm{p}}}{\lambda_{\mathrm{p}}+\lambda_{\mathrm{s} 1}} \mathrm{~W}_{\mathrm{p}}+\frac{\lambda_{\mathrm{s} 1}}{\lambda_{\mathrm{p}}+\lambda_{\mathrm{s} 1}} \mathrm{~W}_{\mathrm{s} 1}$

$$
\mathrm{W}_{\mathrm{s} 1}=\frac{\lambda_{\mathrm{p}}+\lambda_{\mathrm{s} 1}}{\lambda_{\mathrm{s} 1}} \mathrm{~W}_{\mathrm{x} 1}-\frac{\lambda_{\mathrm{p}}}{\lambda_{\mathrm{s} 1}} \mathrm{~W}_{\mathrm{p}}
$$

For class two secondary user: Let $\mathrm{W}_{\mathrm{X} 2}$ be the expected waiting time in the system of a random arrival in either of these primary users, class one secondary users, and class two secondary users:

$$
\begin{aligned}
& \mathrm{W}_{\mathrm{x} 2}=\frac{1}{\left[\mathrm{c} ! \frac{\left(\mathrm{c} \mu-\lambda_{\mathrm{x} 2}\right)}{\left(\frac{\lambda_{\mathrm{x} 2}}{\mu}\right)^{\mathrm{c}}} \sum_{\mathrm{n}=0}^{\mathrm{c}-1} \frac{\left(\frac{\left.\lambda_{\mathrm{x} 2}\right)^{\mathrm{n}}}{\mathrm{n} !}+\mathrm{c} \mu\right]\left[1-\frac{\lambda_{\mathrm{x} 2}}{\mathrm{c} \mu}\right]}{\mu}\right.}+\frac{1}{\mu} \\
& \lambda_{\mathrm{x} 2}=\lambda_{\mathrm{p}}+\lambda_{\mathrm{s} 1}+\lambda_{\mathrm{s} 2} \\
& \mathrm{~W}_{\mathrm{x} 2}=\frac{\lambda_{\mathrm{p}}}{\lambda_{\mathrm{p}}+\lambda_{\mathrm{s} 1}+\lambda_{\mathrm{s} 2}} \mathrm{~W}_{\mathrm{p}}+\frac{\lambda_{\mathrm{s} 1}}{\lambda_{\mathrm{p}}+\lambda_{\mathrm{s} 1}+\lambda_{\mathrm{s} 2}} \mathrm{~W}_{\mathrm{s} 1}+\frac{\lambda_{\mathrm{s} 2}}{\lambda_{\mathrm{p}}+\lambda_{\mathrm{s} 1}+\lambda_{\mathrm{s} 2}} \mathrm{~W}_{\mathrm{s} 2} \\
& \mathrm{~W}_{\mathrm{s} 2}=\frac{\lambda_{\mathrm{p}}+\lambda_{\mathrm{s} 1}+\lambda_{\mathrm{s} 2}}{\lambda_{\mathrm{s} 2}} \mathrm{~W}_{\mathrm{x} 2}-\frac{\lambda_{\mathrm{s} 1}}{\lambda_{\mathrm{s} 2}} \mathrm{~W}_{\mathrm{s} 1}-\frac{\lambda_{\mathrm{p}}}{\lambda_{\mathrm{s} 2}} \mathrm{~W}_{\mathrm{p}}
\end{aligned}
$$

The waiting time for class three can be derived with the same procedures, and the other three parameters can be easily formulated by using equations (4), and (5).

\section{SIMULATION RESULTS}

This section evaluates the proposed model using MATLAB. Results have been carried out by varying the arrival rate of the primary users. The proposed model has been evaluated by four metrics namely, expected waiting time in queue, expected number of users in queue, expected waiting time in the system, and expected number of users in the system. Results have been obtained in the case of five channels.

\subsection{Comparison between The Proposed Model and The Basic Model Queue in [15]}

Authors in [15] provide the description and comparison of 13 structured and simulation modeling systems (SSMS). Structural and simulation modeling systems are compared to each other. Structural model simulated in [15] proposed queuing model without priority. In this subsection, results obtained from the proposed model will be compared with the results in [15]. The parameters used in this simulation are the same as the parameters used in [15].

Multi-Channel Preemptive Priority Model for Spectrum Mobility in Cognitive Radio Networks (S. E. Saad) 
Table 2. Comparison of the Proposed Model with the Basic Model in [15]

\begin{tabular}{|c|c|c|c|c|c|c|}
\hline \multirow[b]{2}{*}{ Parameter } & \multicolumn{5}{|c|}{ Proposed Model } & \multirow[b]{2}{*}{$\begin{array}{c}\text { Results of } \\
{[15]}\end{array}$} \\
\hline & PU & SU1 & SU2 & SU3 & $\begin{array}{c}\text { Total } \\
\text { Network }\end{array}$ & \\
\hline $\mathrm{L}_{\mathrm{q}}$ & 0.0001939 & 0.0084 & 0.0657 & 0.2799 & 0.3541939 & 0.354 \\
\hline $\mathrm{L}$ & 0.7502 & 0.7584 & 0.8157 & 1.0299 & 3.3542 & 3.354 \\
\hline $\mathrm{W}_{\mathrm{q}}$ & 0.0077572 & 0.3374869 & 2.6267 & 11.197 & 3.542236 & 3.542 \\
\hline $\mathrm{W}^{1}$ & 30.008 & 30.337 & 32.627 & 41.197 & 33.54225 & 33.542 \\
\hline
\end{tabular}

As shown in Table 2, total results obtained from the proposed model are the same as the results in [15]. All measured time values in unit time. This simulation has been performed to prove that, the proposed model is validated by comparing the total results achieved with the results of the conventional model.

In I. Yakimov et al a conventional queuing model without priority was presented, which does not meet the requirements for cognitive radio networks [15]. This paper proposes a model for multi-channel, multi-classes cognitive radio networks using the preemptive priority model based on queuing theory. A performance analysis in the most general form can be conducted by the proposed model. In the next subsection, the proposed model will be analyzed to ensure priority for primary users and ensure priority for higher class secondary user over lower class at a different arrival rate.

\subsection{Performance Evaluation of the Proposed Model at Different Request Rate}

In this simulation, the arrival rate of the primary users may vary between 0.01-4 request/sec. In this analysis, the arrival rate of the seconddary users is 4 request/sec for each class, and the service rate is 4 request/sec for each channel.

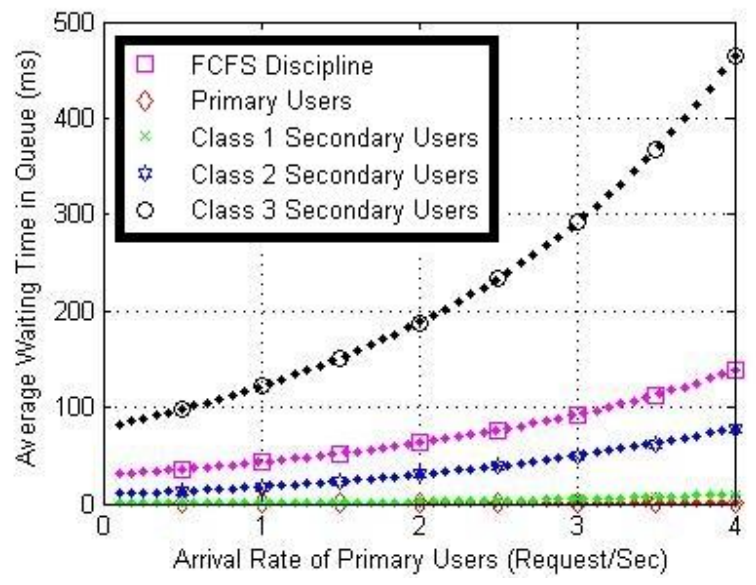

Figure 3. Expected Waiting Time in the Queue

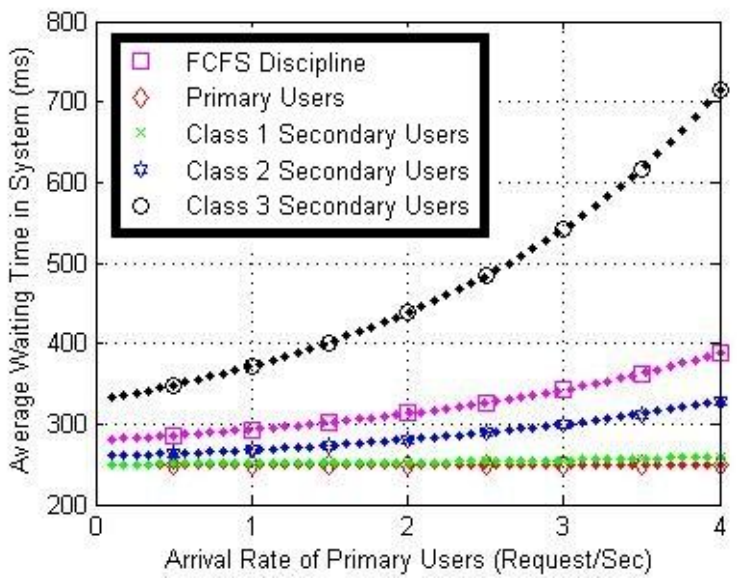

Figure 4. Expected Waiting Time in the System

The performance in terms of waiting time in buffer is shown in Figure 3. It can be observed that, the waiting time in buffer for primary user is the lowest in the system. Results show that, at the point of $\lambda_{\mathrm{p}}=4, \lambda_{\mathrm{s} 1}=\lambda_{\mathrm{s} 2}=$ $\lambda_{\mathrm{s} 3}=4 \mathrm{request} / \mathrm{sec}$; Waiting time for primary and three classes of secondary user is $0.24 \mathrm{~ms}, 9.7 \mathrm{~ms}, 78.6 \mathrm{~ms}$, and $465.6 \mathrm{~ms}$ respectively. We can examine the effect of priority schedule at this point. The waiting time in the queue without priority is $138.5 \mathrm{~ms}$, so, the priority schedule improves the waiting time in queue for class one secondary users, and class two secondary users by $92.99 \%$, and $43.25 \%$ respectively compared to the basic model without priority.

It is important to note that, the average of waiting time for all users of the proposed model at any point is exactly equal to the waiting time in the queue without priority First Come First Serve (FCFS) discipline at the same point, so, the average of waiting time for all users at the point $\lambda \mathrm{p}=4, \lambda_{\mathrm{s} 1}=\lambda_{\mathrm{s} 2}=\lambda_{\mathrm{s} 3}=$ 4 request/sec is $138.5 \mathrm{~ms}$. It is observed that the waiting time in queue increases in an exponential order as the arrival rate of primary users increased. 
Figure 4 shows that, at the point of $\lambda p=4$ request/sec; waiting time for primary and three classes of secondary user is $250.24 \mathrm{~ms}, 259.7 \mathrm{~ms}, 328.6 \mathrm{~ms}$, and $715.6 \mathrm{~ms}$ respectively. To study the impact of priorities, the waiting time in the system without priority is $388.5 \mathrm{~ms}$ so, the priority schedule improves the waiting time in class one secondary users, and class two secondary users by $33.15 \%$, and $15.42 \%$ respectively.

As the primary user arrival rate increases, then due to high priority, the primary user takes the channel from secondary user and the secondary user will be ejected to waiting buffer. Further, as the primary users use more channels in the system, then the waiting time to the secondary users should increase as depicted in Figures 3 and 4. Then, if there isn't an empty channel for secondary user and there is lower priority secondary user in service so; the lower priority secondary user will be ejected to waiting buffer and the higher priority secondary user will be accessed. Consequently, the lower priority secondary user will be more affected by increasing the primary user arrival rate.

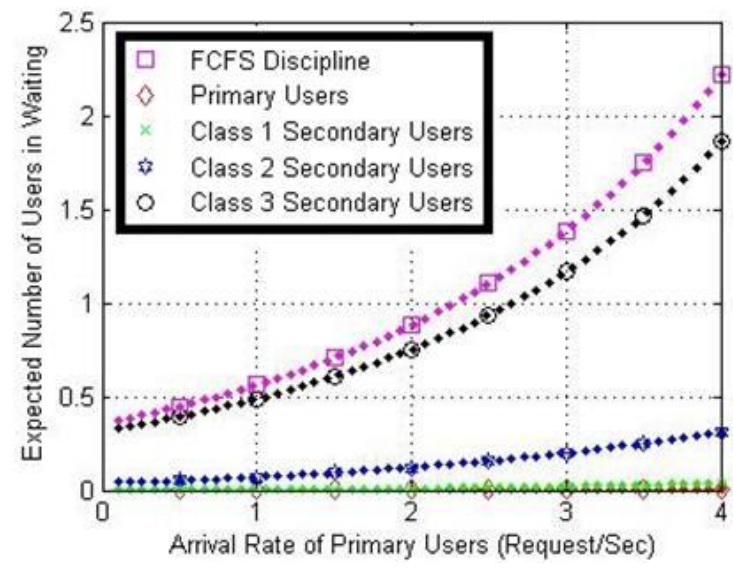

Figure 5. Expected Number of Users in Waiting

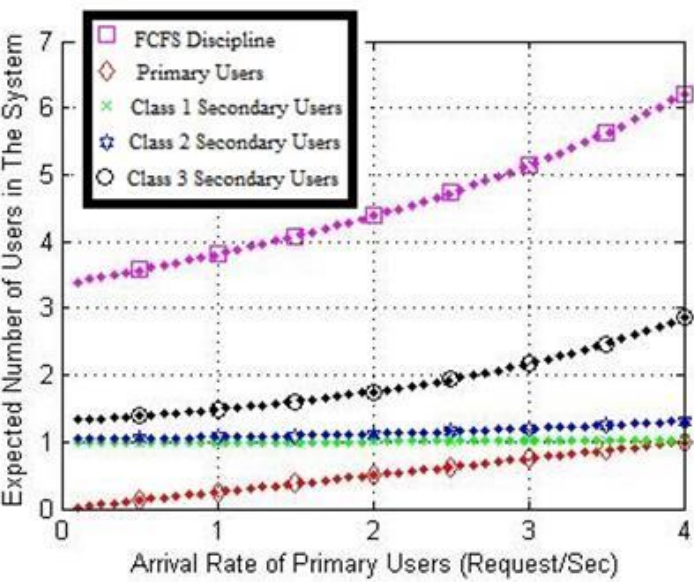

Figure 6. Expected Number of Users in System

As shown in Figure 5, the number of users in the waiting buffer for primary users is lower than others users. Results show that, at the point of $\lambda p=4$ request/sec; number of users in the waiting buffer for primary and three classes of secondary user is $0.00096,0.0388,0.3144$, and 1.86 respectively. To study the impact of priorities, the waiting time in the system without priority is 2.22 so; the priority schedule improves the waiting time in class one secondary users, and class two secondary users by $93 \%$, and $43.35 \%$ respectively.

Figure 6 shows that, the number of users in the system for primary users are lower than others users. Results show that, at the point $\lambda \mathrm{p}=4$ request/sec; number of users in the system for primary and three classes of secondary user is $1.001,1.0388,1.3144$, and 2.8622 respectively. To study the impact of priorities, the number of users in the system without priority is $6.22 \mathrm{so}$; the priority schedule improves the number of users in the system in class one secondary users, and class two secondary users by $33.2 \%$, and $15.47 \%$ respectively.

From the performance analysis results, it is concluded that, at the arrival rate of the primary user increases, the waiting time and the number of users increase, but the priority of each class is reserved. The waiting time for primary users are completely unaffected by the presence of users in the secondary classes.

In most previous studies, the authors consider that the secondary user is a single class. This consideration does not meet the needs of multiple applications for the use of cognitive radio. Therefore, the model presented in this paper is a solution to this problem. Compared with the previous works [8-10, 12], the authors have assumed the cognitive radio networks by assuming the problem as a single channel. However, these models do not adequately overcome the need to analyze of cognitive radio networks as they have multiple channels. Therefore, the model presented in this paper can be efficiently used to analyze the behavior of multi-channel of the priority queue model for cognitive radio networks. 


\section{CONCLUSION}

In this paper, priority based preemptive queuing model has been proposed for channel access in cognitive radio networks. Steady state analysis of the proposed model is presented. Preemption priority is utilized to meet requirements of the class one and class two secondary users. The proposed model gives the commendable schedules of primary and secondary users. This work can be extended to evaluate a preemptive priority discipline in event simulator, and can be extended to analyze the performance in the case of finite size of waiting buffer.

\section{REFERENCES}

[1] O.W. Bello, O.A. Sowande, S.O. Onidare, M.Y. Muhammada, and A.A. Ayeni, "Large scale spectrum survey in rural and urban environments within the $50 \mathrm{MHz}-6 \mathrm{GHz}$ bands", Elsevier, Measurement Journal 91, 2016.

[2] F.Z. El Bahi, H. Ghennioui, and M. Zouak, " Performance Evaluation of Energy Detector Based Spectrum Sensing for Cognitive Radio using NI USRP-2930", International Journal of Electrical and Computer Engineering (IJECE), vol. 7, no. 4, pp. 1934 - 1940, 2017.

[3] A.S. Khobragade1, and R. D. Raut, " Hybrid Spectrum Sensing Method for Cognitive Radio", International Journal of Electrical and Computer Engineering (IJECE), vol. 7, no.5, pp. 2683 - 2695, 2017.

[4] Mohammadreza Amini, Asra Mirzavandi, Mosrafa Rezaei, "Discrete Markov Chain Based Spectrum Sensing for Cognitive Radio", International Journal of Electrical and Computer Engineering (IJECE), vol. 5, no. 2, pp. 297 30, 2015.

[5] S.S. Ali, C. Liu, and M. Jin, "Minimum Eigenvalue Detection for Spectrum Sensing in Cognitive Radio", International Journal of Electrical and Computer Engineering (IJECE), vol. 4, no. 4, p. 623, 2014.

[6] H. Sun, A. Nallanathan, C.X. Wang, and Y. Chen, "Wideband spectrum sensing for cognitive radio networks: a survey", IEEE Wirel. Commun., vol. 20, no. 2, pp. 7481, 2013.

[7] J. Avila, and K. Thenmozhi, "Multiband OFDM for Cognitive Radio - A Way for Cyclostationary Detection and Interference Cancellation”, International Journal of Electrical and Computer Engineering (IJECE), vol. 6, no. 4, pp. $1702-1709,2016$.

[8] L. Chen, L. Huang, H. Xu, and J. Hu, "Queueing Analysis for Preemptive Transmission in Underlay Cognitive Radio Networks", International Journal of Communication Systems, 29: 1138-1155. doi: 10.1002., John Wiley \& Sons, Ltd, 2016.

[9] M.E. Bayrakdar, and, A. Çalhan, "Improving spectrum handoff utilization for prioritized cognitive radio users by exploiting channel bonding with starvation mitigation", Elsevier, International Journal of Electronics and Communications (AË̈), 2017.

[10] M.E. Bayrakdar, and, A. Çalhan, "Non-Preemptive Queueing Model of Spectrum Handoff Scheme based on Prioritized Data Traffic in Cognitive Wireless Networks", ETRI Journal, 39 (4), 558-569, 2017.

[11] T.C. Chu, H. Phan, and H. Zepernick, "Dynamic Spectrum Access for Cognitive Radio NetworksWith Prioritized Traffics", IEEE communications letters, Vol. 18, NO. 7, 2014.

[12] V. Kumar, S. Minz , and Vipin Kumar, "Performance analysis of cognitive radio networks under spectrum sharing using queuing approach", Elsevier, Computers and Electrical Engineering 52, 2016.

[13] Navid Tadayon, "Modeling, Design and Analysis of Multi-Channel Cognitive Radio Networks", PHD Thesis, 2016.

[14] P. Kandasamy, K. Thilagavathi, and K. Gunavathi, "Probability statistics and Queuing theory", S. Chand \&Company LTD., New Delhi, 2006.

[15] I. Yakimov, A. Kirpichnikov, V. Mokshin, Z. Yakhina, and R. Gainullin, "The Comparison of Structured Modeling and Simulation Modeling of Queueing Systems", 16th International Conference, Information Technologies and Mathematical Modelling (ITMM), Queueing Theory and Applications, Springer, 2017. 


\section{BIOGRAPHIES OF AUTHORS}
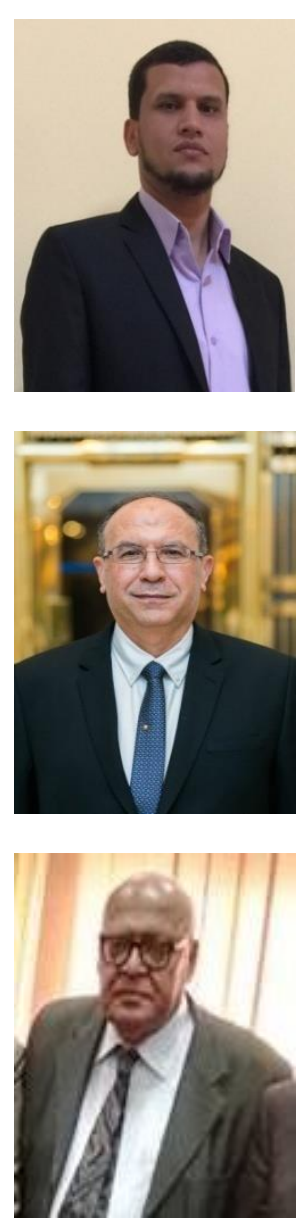

Saad Elsayed is an Assistant Lecturer in the High Institute for Engineering and Technology, AlObour, Cairo, Egypt. He received his BSc and MSc in Electronics and Communications Engineering from the Faculty of Engineering, Al-Azhar University, Cairo, Egypt, in 2008 and 2015 respectively. He is currently a PhD student at Faculty of Engineering, Al-Azhar university, Cairo. His research activities are within wireless communications and communication networks.

Ibrahim Fathy Tarrad received his BSc and MSc degrees in Electronics and Communications Engineering from the Faculty of Engineering, Al-Azhar University, Cairo, Egypt, in 1984 and 1989, respectively. He received his PhD from the Technical University of Budapest in 1996. In 1996, he was appointed Lecturer at the Department of Electronics and Communications Engineering, Al-Azhar University. In 2015, he became an Associate Professor in Communications Engineering at the Faculty of Engineering, Al-Azhar University. His research activities are within wireless communications and digital communications.

Abdelhady Abdelazim Ammar is a professor in Electronics and Communications Engineering department, Faculty of Engineering, Al-Azhar University, Cairo, Egypt since 1988.

His research activities are within digital communications, mobile communications and digital signal processing. 